\title{
Precision Compton polarimetry for Hall C at Jefferson Lab
}

\author{
Donald Jones ${ }^{1}$ \\ University of Virginia \\ Charlottesville, VA, USA \\ E-mail:dcj4v@virginia.edu
}

A new Compton polarimeter was recently built in experimental Hall C at Jefferson Lab in order to meet the strict requirements on knowledge of electron beam polarization needed for the Qweak experiment. This polarimeter, modeled after the Hall A design[1], utilizes a circularly polarized green laser locked to a Fabry-Perot optical cavity intersecting the electron beam at a small angle $\left(\sim 1.3^{\circ}\right)$. Both the scattered electrons and the back-scattered photons are detected separately downstream of the interaction point, providing, in principle, two semi-independent measurements of the electron beam polarization.

I demonstrate the recent performance of the Compton polarimeter with special emphasis on the electron detector analysis. I also discuss a novel technique used to determine the polarization of the light inside the Fabry-Perot cavity with small systematic uncertainty.

XVth International Workshop on Polarized Sources, Targets, and Polarimetry September 9-13, 2013

Charlottesville, Virginia, USA

1

Speaker 


\section{Introduction}

The Qweak[2] experiment took data in Hall C at Jefferson Lab in Newport News, VA from November 2010 to May 2012 with the purpose of measuring the weak charge of the proton. The stringent error budget of this experiment requires a determination of beam polarization with less than $1 \%$ uncertainty. In order to accommodate these demands and those of future experiments, a new Compton polarimeter was built in experimental Hall $\mathrm{C}$ and utilized in the Qweak experiment for the measurement of the electron beam polarization. An existing Møller polarimeter was also used to measure the polarization. The Møller polarimeter utilizes a magnetically saturated iron foil inserted into the beam and can be used accurately at currents $<5 \mu \mathrm{A}$. At higher currents, uncertainty due to foil demagnetization from heating becomes significant. Since Qweak routinely took data at $180 \mu$ A, Møller beam polarization measurements were taken under potentially different beam conditions. The Compton polarimeter has the advantages of being non-invasive, continuous and not limited by high beam current. Although the high currents used in Qweak were optimal for Compton polarimetry, the low beam energies $(\sim 1.16 \mathrm{GeV})$ present the challenge of a small analyzing power for the Compton scattering asymmetry.

The longitudinal polarization of the electron beam is determined by measuring the asymmetry of scattering rates between positive and negative helicity electron beam states with a specific laser helicity state as seen in the equation below:

$$
A_{\text {Comp }}=\frac{\sigma_{R}-\sigma_{L}}{\sigma_{R}+\sigma_{L}}
$$

where $\sigma_{R(L)}$ are the scattering cross sections for right(left) handed helicity electrons. The electron beam polarization is then given as $A_{\text {meas }}=P_{\gamma} P_{e} A_{\text {Compton }}$, where $P_{\gamma}$ is the laser polarization, $P_{e}$ is the electron beam polarization and $A_{\text {Compton }}$ is the theoretical Compton scattering asymmetry. The theoretical asymmetry for the kinematics used in Qweak (electron energy $1.16 \mathrm{GeV}$ and photon wavelength $532 \mathrm{~nm}$ ) is shown in Figure 1. 


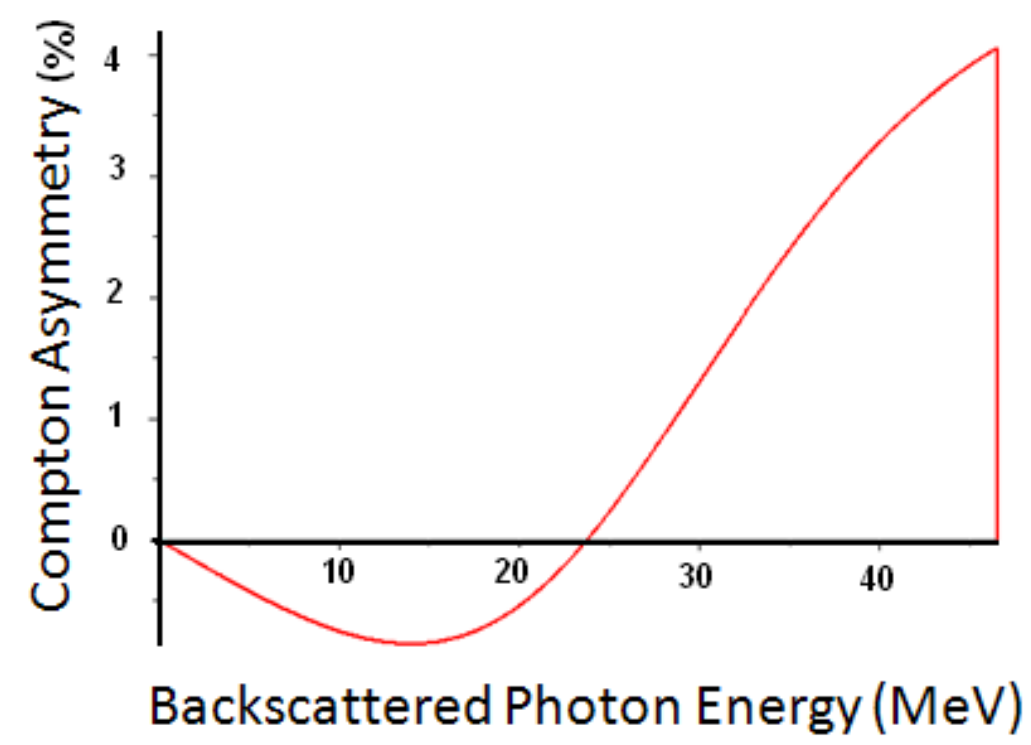

Figure 1. Theoretical Compton scattering cross section asymmetry of left and right helicity electrons from photons of parallel and anti-parallel spins. The "Compton edge"(kinematic maximum) at $47 \mathrm{MeV}$ is shown as a vertical line in the figure.

\subsection{Overview}

Figure 2 shows a schematic of the Compton polarimeter in Hall C. The electron beam is bent through a magnetic chicane of four identical dipole magnets wired in series to ensure the total integrated $\vec{B} \bullet d \vec{l}$ is zero. An optics table located between the second and third dipoles houses the laser and associated optics for producing the photon target. A $10 \mathrm{~W}$ Coherent Verdi ${ }^{\mathrm{TM}}$ [3] green laser $(532 \mathrm{~nm})$ is locked to a medium gain ( 200) external Fabry-Perot optical cavity giving $>1500 \mathrm{~W}$ of stored laser power in the cavity. The laser is changed from linearly to circularly polarized before entering the cavity with a linear polarizer followed by a quarter wave plate (QWP). A half-wave plate (HWP) inserted in the beam just before the QWP allows the laser helicity (left or right handed) to be selected. The electron beam intersects the laser at the center of the optical cavity. The electron beam optics are set up to create a waist in the electron beam radius at the point of intersection with the laser. The electron beam has a Gaussian width of approximately $40 \mu \mathrm{m}$ at the point of intersection with the Compton laser. The laser was focused to a much larger $180 \mu \mathrm{m}$ waist to ensure the full electron beam was sampled. The backscattered photons are detected in a lead tungstate detector located on the downstream side of the third dipole. The third dipole acts as a spectrometer for bending the scattered electrons off the main beam trajectory onto the diamond strip electron detector. 


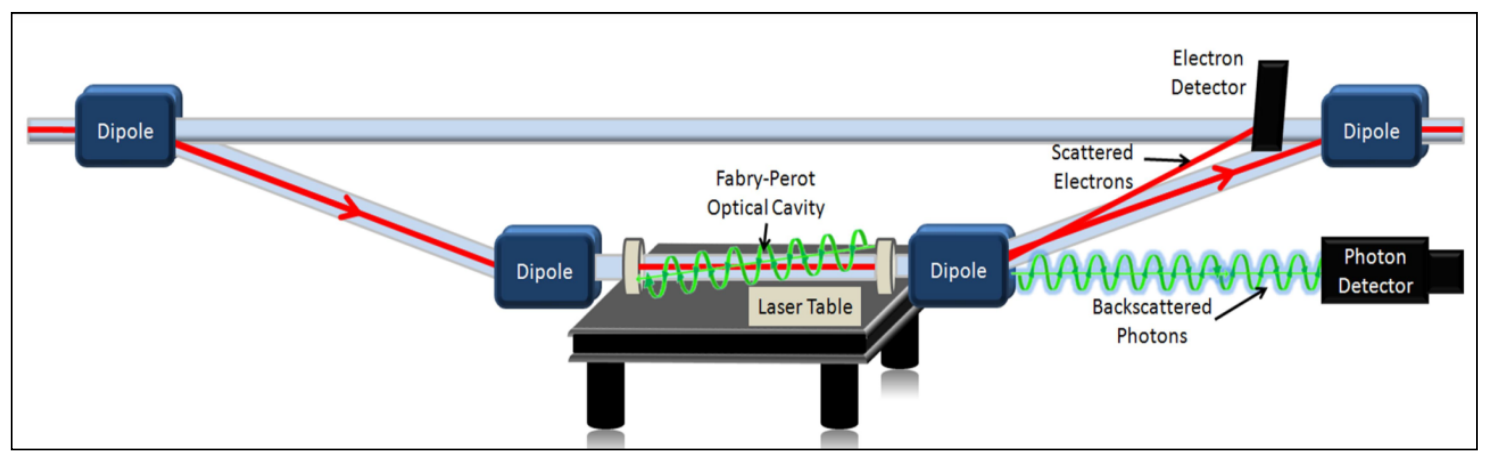

Figure 2. Schematic of Compton polarimeter hardware. The electron beam is bent through a magnetic chicane of four dipole magnets. The electron beam interacts with a laser locked to a Fabry-Perot optical cavity between the second and third dipoles.

\section{Subsystems and Analysis}

The Compton polarimeter is composed of three main subsystems. First, the laser system produces the circularly polarized photon target. Uncertainties arising from this subsystem, such as laser polarization, are common to both the electron and photon detectors. Second, the electron detector proper along with its readout chain. Third, the photon detector along with its electronics readout chain. The third dipole magnet in the chicane is also a part of both the electron and photon detectors acting to separate the scattered electrons both from the backscattered photons and from the unscattered electrons.

\subsection{Laser System}

The photon target is composed of a 10W Coherent Verdi ${ }^{\mathrm{TM}}$ [3] laser locked to a FabryPerot optical cavity with a gain of approximately 200. The cavity is $84 \mathrm{~cm}$ long crossing the electron beam at a $1.3^{\circ}$ angle. The low-loss cavity mirrors (provided by Advanced Thin Films, Boulder, CO) have a reflectivity of $99.5 \%$ and a radius of curvature of $50 \mathrm{~cm}$. The laser is very close to an ideal Gaussian beam with quality parameter $\mathrm{M}^{2}<1.1$. The Verdi laser head was retrofitted by Coherent with PZT-actuated mirrors on the internal cavity to allow for feedback on laser wavelength. The light is shaped by a series of lenses on the optics table to match it to the $\mathrm{TEM}_{00}$ mode of the optical cavity. For purposes of electron beam polarimetry the laser must be circularly polarized. A circularly polarized beam is created by passing the laser through a linear polarizer followed by a quarter-wave plate. A half-wave plate (HWP) is also inserted into the laser after the linear polarizer in order to select the laser helicity. This combination of linear polarizer, HWP and QWP allows any arbitrary polarization and helicity state to be selected. A simplified schematic of the setup is shown in Figure 3.

Given the a mirror reflectivity of $\mathrm{R}=0.995$ and the cavity length $0.84 \mathrm{~m}$, the finesse of the optical cavity can calculated to be $F=630$ and the free spectral range $f_{s r}=180 \mathrm{MHz}$. The cavity linewidth, which is approximately equal to the ratio of the free spectral range to the finesse, is $290 \mathrm{kHz}$. There was initial concern in the design of the laser system that the intrinsic bandwidth of the laser would be too wide to maintain a clean cavity lock. Coherent ${ }^{\mathrm{TM}}$ specifications give the bandwidth of the laser as $<5$ 
$\mathrm{MHz}$ over $50 \mathrm{~ms}$. However, the bandwidth of the laser was found to be sufficiently narrow over shorter timescales to allow for a clean lock.

In order for light to build up in the optical cavity, the roundtrip distance must be an integer number of wavelengths of the laser light. This condition is stated mathematically as $n \lambda=2 L$, where $\mathrm{L}$ is the cavity length. When this condition is maintained, the cavity is said to be "locked" and light will build up between the cavity mirrors. The cavity is locked using the Pound-Drever-Hall (PDH)[4] locking technique feeding back on the laser wavelength. The PDH prescription utilizes an electro-optical modulator to EOM to modulate the laser frequency. In our case the modulation frequency is $6 \mathrm{MHz}$. The light reflected from the cavity mirror is monitored using an amplified photodiode connected to an integrating sphere. This reflected photodiode signal is mixed with the modulation frequency and passed through a low pass filter to produce a feedback signal approximately proportional (over a small frequency range centered on cavity resonant frequency) to the difference between laser frequency and the resonant frequency of the cavity. A subsequent servo loop used to maintain the cavity on-resonance was incorporated in a single electronics module (Digilock ${ }^{\mathrm{TM}}[5]$ ) purchased from Toptica $^{\mathrm{TM}}$ Photonics.

If perfectly circularly polarized light is produced and arrives at the cavity, the reflected light will have the reverse helicity and be blocked by the linear polarizer thus being measured in the reflection monitor. Residual linear polarization will not be completely blocked by the linear polarizer and part of it will travel back to the laser head. This "leakage" light is monitored in the leakage monitor. Minimizing the leaked light maximizes the degree of circular polarization (DOCP) at the cavity. This technique was verified by direct measurement of laser linear polarization in the cavity region and calculating circular polarization as $D O C P=\sqrt{1-P_{\text {linear }}^{2}}$, under the assumption that the laser has no unpolarized component. We were able to bound the unpolarized fraction of the laser light to $<0.04 \%$ by direct measurement. Using this technique of minimizing leakage light we were able to achieve 100(-0.2)\% DOCP for most of the Qweak experiment.

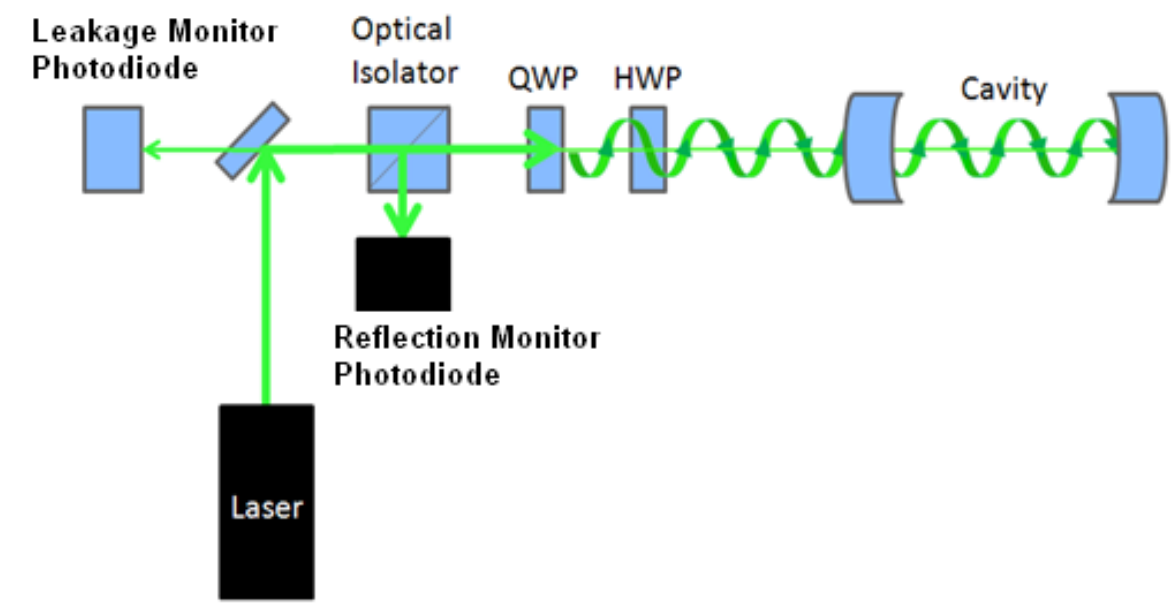

Figure 3. Simplified schematic showing the key components of the polarization state generator and reflected light monitors. 


\subsection{Electron Detector}

The electron detector[6][7] consists of four $0.5 \mathrm{~mm} \times 21 \mathrm{~mm} \times 21 \mathrm{~mm}$ diamond planes. Each plane has 96 metal microstrips deposited on the top surface and a single electrode deposited on the bottom surface. Each microstrip is 180 microns wide with a space of 20 microns between adjacent strips. A negative high voltage was applied between the two surfaces across the thickness of the diamond plate with the top surface at zero potential. When an electron enters the diamond strip it creates electron-hole pairs. The electrons migrate toward the top surface creating a pulse on the nearest microstrip. This pulse is then conditioned and shaped before being analyzed by the trigger logic. When an electron track is formed, all the microstrips are read out and stored. For a signal to be considered a valid track, there must be a signal in two or more planes of the same $( \pm 2)$ microstrip number. The integrated number of hits for the two electron beam helicities is determined on a run by run basis and an asymmetry formed for each strip as follows:

$$
A_{\text {strip }}=\frac{\left(N_{\text {strip }}^{R}-N_{\text {strip }}^{L}\right)}{\left(N_{\text {strip }}^{R}+N_{\text {strip }}^{L}\right)},
$$

where $N_{\text {strip }}^{R(L)}$ is the integrated number of tracks for the right (left) electron beam helicity states for a given strip. The number of counts, $N_{\text {strip }}^{R(L)}$, is background subtracted by alternately measuring rates with the laser on and off and taking the time normailzed difference as shown in the following formula: $N=N_{\text {on }}-N_{\text {off }}\left(t_{\text {on }} / t_{\text {off }}\right)$. An example of such an asymmetry spectrum in shown in Figure 5. This strip by strip asymmetry gives an asymmetry spectrum as a function of distance from the beam and thus, effectively, of electron energy. This asymmetry spectrum is fit to a functional form of the theoretical Compton scattering asymmetry (see Figure 1) with two fit parameters, the energy of the kinematic maximum (Compton edge) and the beam polarization. Figure 6 shows the polarization results given by the electron detector for a period of about four months during the Qweak experiment. Polarization results for the Møller polarimeter are also shown on the same plot. The two polarimeters show decent agreement. 


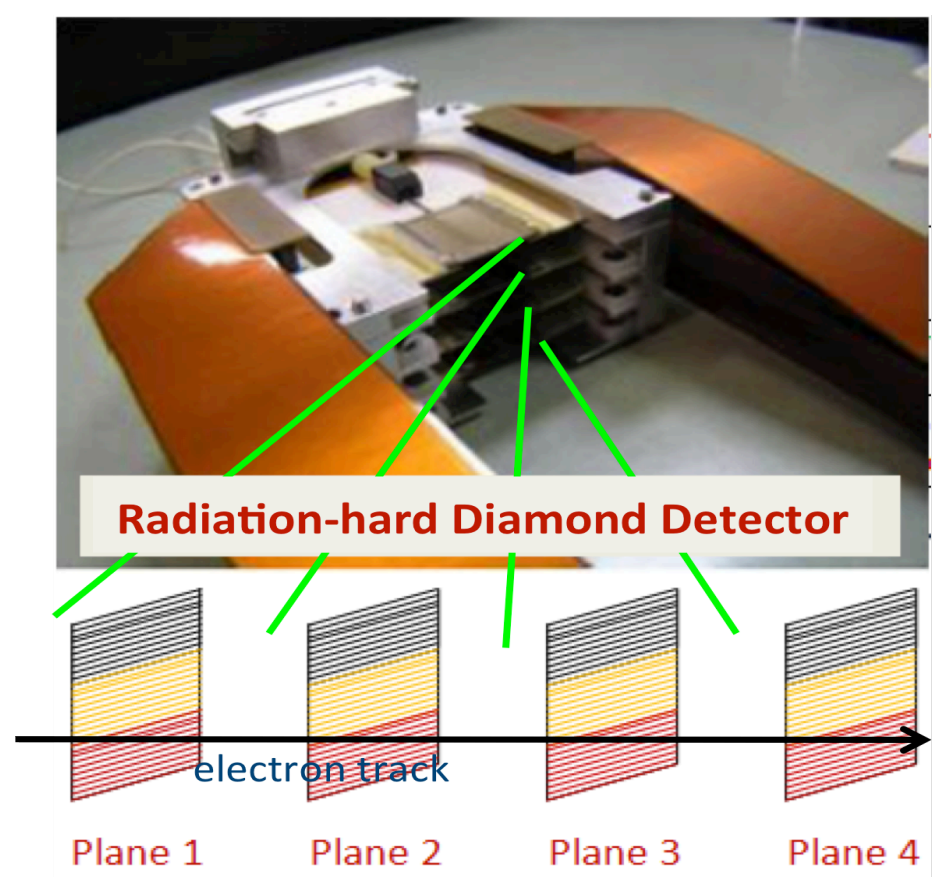

Figure 4. Picture of four electron detector diamond planes each having 96 microstrips. A signal is considered a track if microstrips of the same number $( \pm 2)$ fire in two or more planes simultaneously.

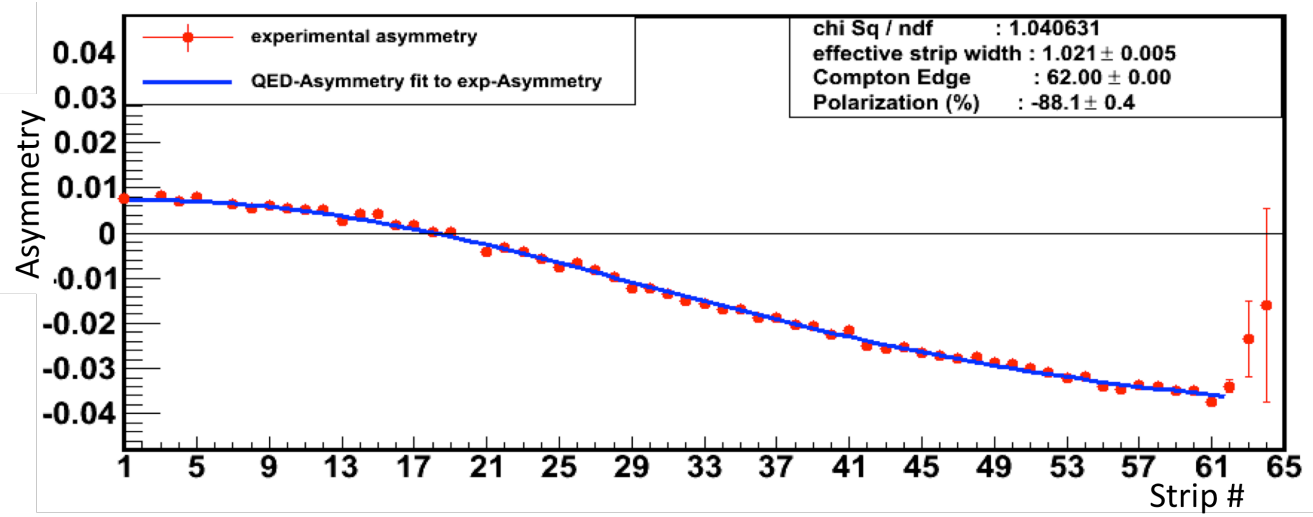

Figure 5. Electron detector asymmetry as a function of strip number. Distance from unscattered electron beam increases with strip number. The kinematic maximum is obvious at strip 62. 


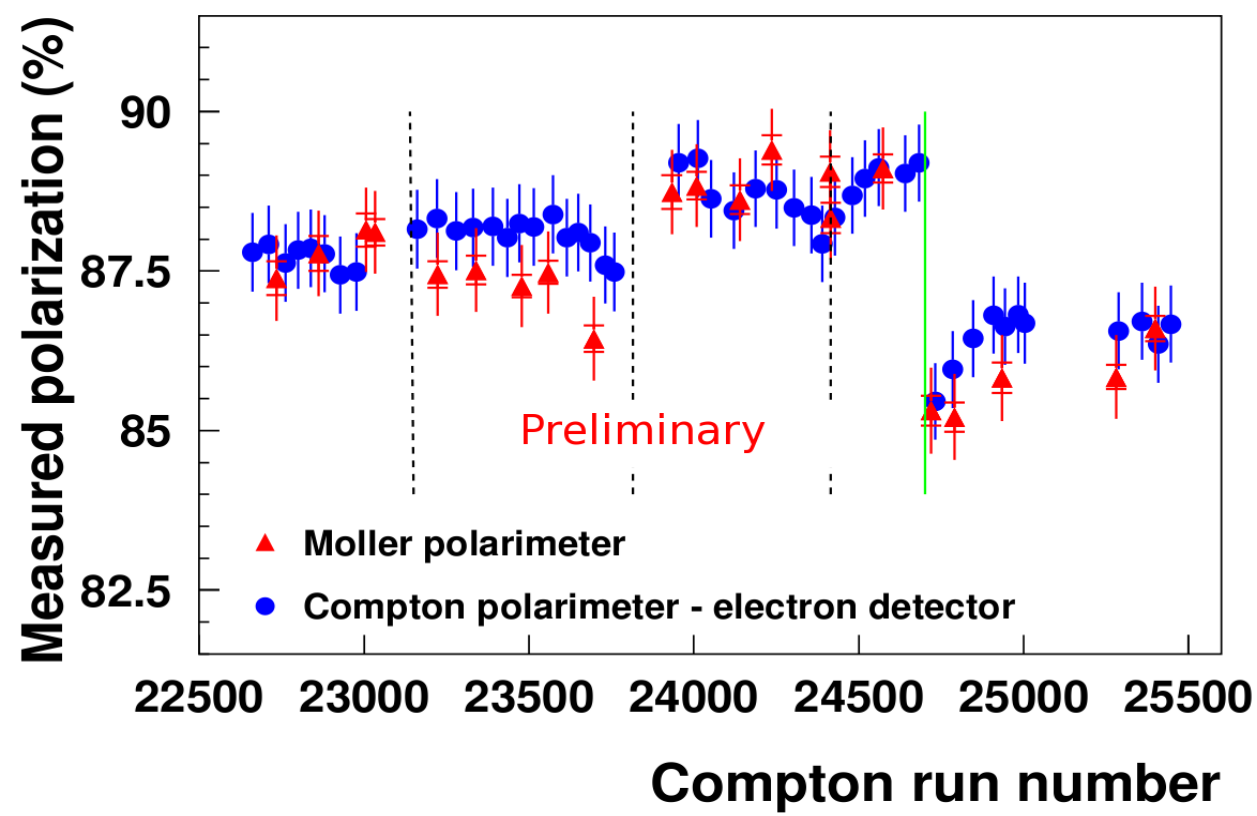

Figure 6. Electron detector polarization measurements taken over a four month period during the Qweak experiment. Also shown is the electron beam polarization results for the Møller polarimeter over the same period. Møller uncertainties include statistical and point to point systematic errors added in quadrature.

\subsection{Photon Detector}

The photon detector, located downstream of the third dipole, is composed of a $2 \times 2$ matrix of $\mathrm{PbWO}_{4}$ crystals each measuring $3 \mathrm{~cm} \times 3 \mathrm{~cm} \times 21 \mathrm{~cm}$. A single 3 inch photomultiplier tube (PMT) is used to collect the light. Rather than recording individual pulses in the photon detector and creating an asymmetry spectrum as in the case of the electron detector, the signal from photon detector is integrated over each helicity window. This measurement is an energy-weighted spectrum integrated over the full range of scattered photon energies from 0 to the kinematic maximum at the Compton edge. The background event spectrum must be accurately measured by turning the laser off and then subtracted from the measured spectrum. For the Qweak experiment the laser was continuously cycled with the laser on for 60 seconds and off for 30 seconds. The average of the background measured during the 30 seconds before and after a 60 second period was subtracted from each laser on period. For the kinematics of the Qweak experiment, the integrated, energy weighted asymmetry is about $2 \%$.

In order to obtain electron beam polarization results from photon detector asymmetries, the detector non-linearity must be determined and a precise analyzing power for the $\mathrm{PbWO}_{4}$ crystals obtained from simulation. Efforts to obtain these are ongoing. Raw asymmetry results for the photon detector are shown in Figure 7. 


\section{Laser On Asymmetry vs Run}

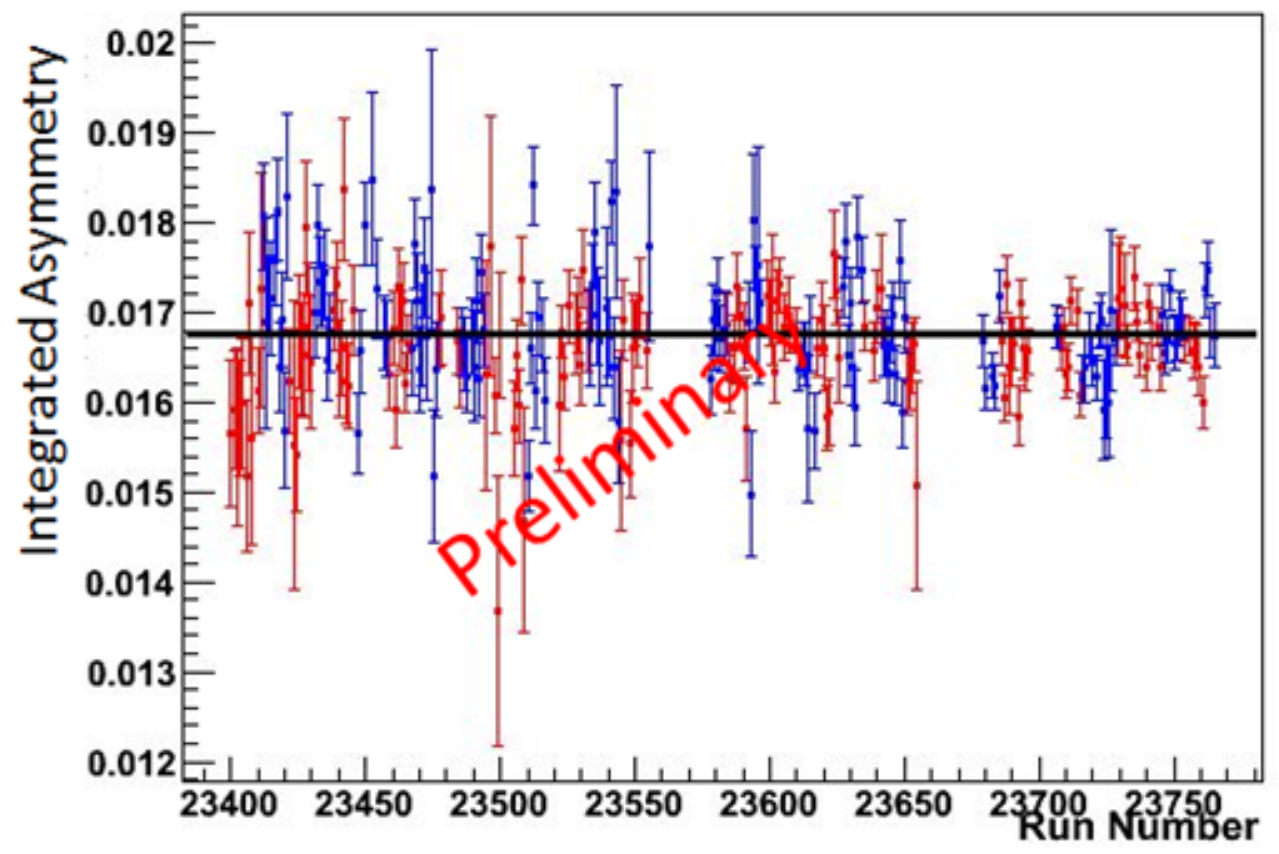

Figure 7. Photon detector asymmetries measued during the Qweak experiment.

\section{Conclusion}

A new Compton polarimeter has been commissioned in Hall $\mathrm{C}$ during the Qweak experiment with very promising preliminary results from the electron detector in good agreement with measurements from the existing Møller polarimeter over the same time period. Efforts to obtain polarizations from the photon detector asymmetries are underway. A new method for determining laser polarization, a key systematic error in these measurements, has allowed a precise determination of the degree of circular polarization.

\section{References}

[1] G. Bardin et.al.,A Conceptual Design Report of a Compton Polarimeter for Cebaf Hall A

[2] D. Androic et al. (Qweak Collaboration), First Determination of the Weak Charge of the Proton, Phys. Rev. Lett. 111, 141803(2013)

[3] Operator's Manual Verdi ${ }^{T M} V-8 / V-10$ Diode-Pumped Lasers. C) Coherent, Inc. 08/2005, Part No. 0174-929-00, Rev DB

[4] Eric D. Black, An Introduction to Pound-Drever-Hall laser frequency stabilization [DOI: 10.1119/1.1286663]

[5] Digilock 110 Feedback Controlyzer Manual. Manual M-031 Version 04 Copyright $(2009$ Toptica Photonics AG. 
[6] A. Narayan, D. Dutta, V. Tvaskis, and J. Martin, A diamond micro-strip electron detector for Compton polarimetry, Nuovo Cimento C vol 035, No. 4 (2012), pp 134;

[7] J. W. Martin, D. Dutta, D., A. Narayan, and P. Wang, AIP Conf. Proc. 1182, 623 (2009)

\section{Acknowledgements}

The hardware and data presented have been the cumulative work of the Hall C Compton polarimetry group at Jefferson Lab including personnel and resources from several institutions and has been funded by the Department of Energy and NSERC.

Contributors:

Jefferson Lab, D. Gaskell and the Hall C staff; College of William and Mary, W. Deconinck, J. C. Cornejo; Mississippi State University, D. Dutta , A. Narayan; MITBates, S. Kowalski, E.Ihloff, and technical staff; University of Manitoba, V. Tvaskis; University of Virginia, M. Dalton, K. Paschke, D. Jones; University of Winnipeg/ TRIUMF, J. Martin , L. Lee, D. Ramsay, L. Kurchaninov. 